\title{
Polarization splitting directional coupler using tilted subwavelength gratings
}

\author{
José Manuel luque-González, ${ }^{1, *}$ Alaine Herrero-Bermello, ${ }^{2,+}$ Alejandro \\ Ortega-Moñux, ${ }^{1}$ Marina Sánchez-Rodríguez, ${ }^{1}$ Aitor V. Velasco, ${ }^{2}$ Jens H. \\ SChmid, ${ }^{3}$ Pavel Cheben, ${ }^{3}$ Í̃̃ Igo Molina-Fernández, ${ }^{1,4}$ and Robert HaliR ${ }^{1,4}$ \\ ${ }^{1}$ Universidad de Málaga, Dpto. de Ingeniería de Comunicaciones, ETSI Telecomunicación, Campus de Teatinos s/n, 29071 Málaga, Spain \\ ${ }^{2}$ Institute of Optics, Spanish National Research Council, 28006 Madrid, Spain \\ ${ }^{3}$ National Research Council Canada, 1200 Montreal Road, Bldg. M50, Ottawa K1A OR6, Canada \\ ${ }^{4}$ Bionand Center for Nanomedicine and Biotechnology, Parque Tecnológico de Andalucía, 29590 Málaga, Spain \\ *Corresponding author:jmlg@uma.es \\ ${ }^{+}$Currently with Alcyon Photonics S.L., Madrid 28006, España
}

Received XX Month XXXX; revised XX Month, XXXX; accepted XX Month XXXX; posted XX Month XXXX (Doc. ID XXXXX); published XX Month XXXX

On-chip polarization splitters are key elements for coherent optical communication systems and polarization diversity circuits. These devices are often implemented with directional couplers that are symmetric for one polarization and strongly asymmetric for the other polarization. To achieve this asymmetry, highly dissimilar waveguides are used in each coupler arm, often requiring additional material layers or etch steps. Here we demonstrate polarization splitting with a directional coupler composed of two fully etched subwavelength waveguides, which only differ in the tilt angle of the silicon segments. Our device exhibits deepUV compatible feature sizes, is only $14 \mu \mathrm{m}$ long, and covers a $72 \mathrm{~nm}$ bandwidth with insertion losses below 1 $\mathrm{dB}$ and an extinction ratio in excess of $15 \mathrm{~dB}$.

http://dx.doi.org/XX.XXXX/OL.XX.XXXXX

Silicon-on-Insulator (SOI) offers multiple benefits as a photonics platform, including CMOS compatible fabrication, and a high index contrast that enables dense integration [1]. The high contrast enables waveguiding at sub-micron scale and, consequently, very high integration densities, albeit at the cost of a high polarization dependence, which is why polarization diversity schemes are required for many applications [2-5]. While larger micron-scale waveguides can significantly reduce this polarization dependence [6,7], they do so at the expense of integration density. Polarization beam splitters (PBS) are key building blocks for onchip polarization diversity architectures. For applications in optical communications, such as polarization multiplexed coherent receivers, $\mathrm{PBS}$ with an extinction ratio of $16 \mathrm{~dB}$ are deemed almost ideal as digital signal processing is used at the receiver end. Moreover, insertion losses have to be kept as low as possible [5].
PBS can be implemented with a variety of approaches, including 1D or 2D grating couplers [8,9], multimode interferometers [10], slotted waveguides [11], mode evolution based devices [12,13] or devices designed via inverse/non-linear algorithms [14,15]. Among those approaches, mode evolution based PBS traditionally show the better performance at expense of relatively large footprints. On the contrary, inverse/non-linear algorithm designs achieve extremely short devices at expense of a complex design process and a limited low-loss bandwidth. However, arguably the most widely used mechanism for polarization splitting in integrated photonic chips is directional coupling (DC-PBS) [16-26]. These splitters can be classified in two categories, symmetric and asymmetric. Symmetric DC-PBS comprise two identical coupled waveguides where both polarizations satisfy the phase matching condition. The device length $L$ is designed to verify $L=m L_{\pi}^{\mathrm{TE}}=n L_{\pi}^{\mathrm{TM}}$, where $L_{\pi}$ is the coupling length of the TE/TM modes, and $m$ and $n$ are integers with $|m-n|$ being an odd number. However, symmetric DC-PBS are often long because the above condition usually requires high values for $m$ and $n$. Moreover, the operational bandwidth and robustness of symmetric DC-PBS is limited because they exhibit a wavelength dependent behavior for both polarizations caused by the narrowband behavior of the phase matching condition. On the contrary, in an asymmetric DC-PBS only one of the polarizations satisfies the phase matching condition, thus coupling to the second waveguide, while the other polarization remains in the original waveguide. The device length is then optimized for the polarization that couples to the second waveguide, while the other polarization does not impose additional restrictions. As a result, asymmetric configurations are typically more compact and exhibit a better performance and tolerance to fabrication errors. On the downside, achieving the required asymmetry complicates the design and fabrication process, requiring additional materials [17,22,25], or two etch steps [26]. 


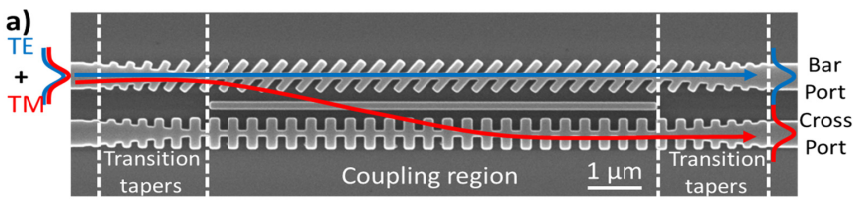

b)

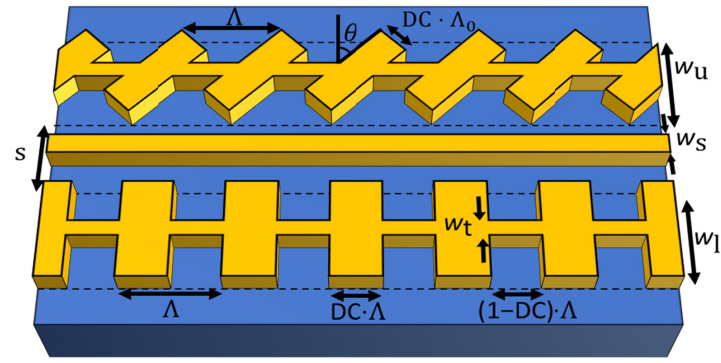

Fig. 1. (a) Scanning electron image (SEM) of the directional coupler based polarization beam splitter (before $\mathrm{SiO}_{2}$ cladding deposition). The tilted SWG segments in the top waveguide break the structural symmetry for the TE mode, which remains in this waveguide. The TM mode is unaffected by the tilt and couples to the cross port. (b) Schematic of the coupling region of the polarization beam splitter (the cladding is not shown for the sake of clarity).

Subwavelength metamaterials, which can be fabricated in a single etch step [27-29], offer the promising capability to implement lithographically tailorable metamaterials modeled as homogeneous uniaxial crystals [30]. These structures can be exploited to implement asymmetric directional couplers by combining conventional and subwavelength waveguides [31,32]. Recently it has been shown that tilting the subwavelength segments significantly changes the propagation constant of TE polarized modes [33,34], but does not substantially affect the TM modes. In this paper we leverage this property to demonstrate for the first time a high-performance asymmetric DC-PBS based on two subwavelength waveguides which only differs in the tilting angle of the silicon segments, as shown in Fig. 1(a). This tilt breaks the phase matching for TE polarization, without affecting the cross-coupling for TM polarization. Our device exhibits a compact footprint of $2 \times 14 \mu \mathrm{m}^{2}$. The measurements demonstrate a $72 \mathrm{~nm}$ bandwidth with a polarization extinction ratio in excess of $15 \mathrm{~dB}$ and, critically, sub-decibel insertion loss. Our device, shown in Fig. 1(b), is based on two subwavelength grating (SWG) waveguides separated by a distance $s=0.5 \mu \mathrm{m}$, to allow power coupling between them. The strip between the arms is a performance optimization that is detailed below. The SWG waveguide widths are initially set to $w_{\mathrm{u}}=w_{\mathrm{l}}=0.6 \mu \mathrm{m}$ to sufficiently confine the field, thus avoiding leakage losses [35], while being insensible to fabrication jitter [36]. The period of the structure is $\Lambda_{0}=275 \mathrm{~nm}$ when $\theta=0^{\circ}$, with a duty cycle $\mathrm{DC}=0.5$ to facilitate fabrication. The tip width $w_{\mathrm{t}}=100 \mathrm{~nm}$ provides a smooth transition between the input waveguide and the SWG structure. In this case, we have chosen a typical $100 \mathrm{~nm}$ tip, however, this value could be slightly increased to facilitate fabrication but would result in a longer device. The grating segments of the upper waveguide are tilted by an angle, $\theta$. We enlarge the period of both structures to $\Lambda=\Lambda_{0} / \cos (\theta)$, thereby maintaining the distance between the SWG segments. The smallest feature of the device is $w_{\mathrm{t}}$ at the homogeneous-to-periodic taper tip. We use a standard 220-nmthick silicon layer with a silicon dioxide top cladding with refractive indexes $n_{\mathrm{Si}}=3.476, n_{\mathrm{SiO} 2}=1.444$, at a central wavelength of $\lambda_{0}=1.55 \mu \mathrm{m}$. To illustrate the principle of operation of our device, we first consider a simplified structure
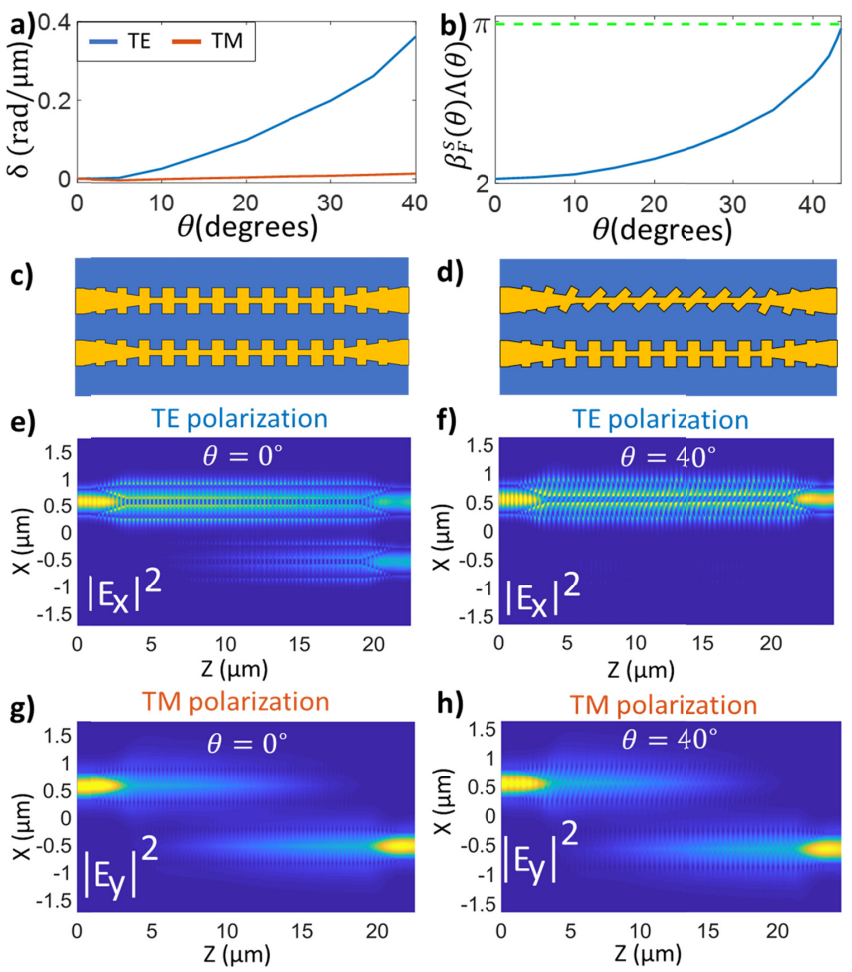

Fig. 2. (a) TE and TM phase matching detuning between the upper and lower waveguides with respect to the tilt angle $\theta$. (b) Phase length of a period as a function of the tilt angle for the fundamental TE supermode. The Bragg condition is reached when $\beta_{F}^{S}(\theta) \cdot \Lambda(\theta)=\pi$, where $\beta_{F}^{S}$ is the phase constant of the fundamental TE supermode and $\Lambda(\theta)=\Lambda_{0} / \cos (\theta)$. (c), (d) Schematic representation of the proposed directional coupler for a tilt angle of (c) $\theta=0^{\circ}$ and (d) $\theta=40^{\circ}$. (e), (f), (g), (h) 3D-FDTD simulations of the squared magnitude of the main component of the propagating electric field for (e), (f) TE and (g), (h) TM polarization for a tilt angle of (e), (g) $\theta=0^{\circ}$ and (f), (h) $\theta=40^{\circ}$.

without the silicon strip between the directional coupler arms [See Fig. 2(c) and Fig. 2(d)]. According to coupled mode theory [37], in a directional coupler the maximum power transferred to the cross port is inversely proportional to the detuning $\delta$ between the effective indexes of the modes of the individual waveguides with $\delta=\pi \Delta n_{\text {eff }} / \lambda$. Figure 2(a) shows the detuning between the waveguides as a function of the tilt angle for both TE and TM polarization. The effective indexes of the modes were calculated using 3D-FDTD simulations [38], following the procedure outlined in Ref. [39]. As expected, increasing the tilt angle results in a strong detuning for the fundamental TE modes, while the effect on the fundamental TM modes is approximately 25 times smaller for the $40^{\circ}$ tilt structure. While higher tilt angles would result in higher detuning between the individual TE waveguide modes, the fundamental TE supermode reaches the Bragg condition $\beta_{F}^{S}(\theta) \Lambda(\theta)=\pi$ for a tilt angle of $\theta \sim 45^{\circ}$ [See Fig. 2(b)], due to the effective increase of the period with the tilt angle. Here $\beta_{F}^{s}$ is the phase constant of the fundamental TE supermode. We compensate the minor detuning for TM polarization at a $40^{\circ}$ tilt angle by slightly changing the width of the lower waveguide from $0.6 \mu \mathrm{m}$ to $0.65 \mu \mathrm{m}$. The effect of the asymmetry introduced by the tilt is illustrated in Figs. 2(e), (f) and Figs. 2(g), (h) for tilt angles $\theta=0^{\circ}$ and $\theta=40^{\circ}$. The propagation of the electromagnetic field reveals that while TM polarization completely couples to the cross port in both cases, the $40^{\circ}$ tilt helps completely isolate the waveguides for TE polarization. 

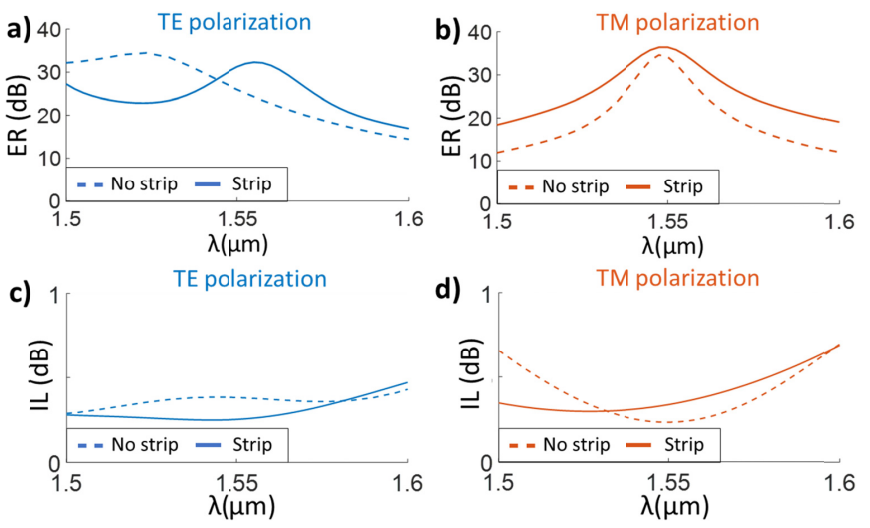

Fig. 3. Simulated extinction ratio for the (a) TE and (b) TM polarizations, and insertion loss for the (c) TE and (d) TM polarizations, of the tilted SWG directional coupler with (solid line) and without (dashed lines) the central silicon strip.

To evaluate the performance of this preliminary design, we use the insertion loss and extinction ratio, defined as:

$$
\begin{aligned}
\mathrm{IL}^{\mathrm{TE}}=\mathrm{P}_{\text {bar }}^{\mathrm{TE}} / \mathrm{P}_{\text {in }}^{\mathrm{TE}} ; & \mathrm{IL}^{\mathrm{TM}}=\mathrm{P}_{\text {cross }}^{\mathrm{TM}} / \mathrm{P}_{\mathrm{in}}^{\mathrm{TM}} ; \\
\mathrm{ER}^{\mathrm{TE}}=\mathrm{P}_{\text {cross }}^{\mathrm{TE}} / \mathrm{P}_{\text {bar }}^{\mathrm{TE}} ; & \mathrm{ER}^{\mathrm{TM}}=\mathrm{P}_{\text {bar }}^{\mathrm{TM}} / \mathrm{P}_{\text {cross }}^{\mathrm{TM}} ;
\end{aligned}
$$

where $\mathrm{P}_{\mathrm{bar}}^{\mathrm{TE} / \mathrm{TM}}$ and $\mathrm{P}_{\text {cross }}^{\mathrm{TE} / \mathrm{TM}}$ is the power in the fundamental $\mathrm{TE} / \mathrm{TM}$ mode in the bar and cross ports, and $\mathrm{P}_{\mathrm{in}}^{\mathrm{TE} / \mathrm{TM}}$ is the power of the fundamental TE/TM mode the input port. To calculate these parameters, we analyze the structure with 3D-FDTD simulations for different wavelengths. The dashed lines in Fig. 3 show the simulated IL and ER of the tilted SWG directional coupler, achieving an ER $>20 \mathrm{~dB}$ over a $40 \mathrm{~nm}$ bandwidth with negligible $\mathrm{IL}$, being mainly limited by the wavelength dependent phase matching condition for TM polarization.

To improve the bandwidth of the device, we introduce the central silicon strip (see Fig. 1), transforming the device into a bridged directional coupler $[32,40,41]$. From an intuitive point of view, this silicon central strip only affects the even TM supermode, thereby allowing us to engineer the coupling length, defined as:

$$
L_{\pi}^{T M}(\lambda)=\frac{\lambda}{2\left[n_{1}^{T M}(\lambda)-n_{2}^{T M}(\lambda)\right]^{\prime}}
$$

where $\lambda$ is the free-space wavelength and $n_{1,2}^{T M}$ are the effective indices of the even and the odd TM polarized supermodes. In Fig. 4(a) we show the resulting coupling lengths for TM polarization for the proposed directional coupler with a tilt angle of $40^{\circ}$ when we change the width of the silicon strip. It is observed that as the strip is widened, the coupling length is reduced. Moreover, as the variation of the beat length with wavelength is, to first order, proportional to the square of the beat length, the device also becomes less wavelength dependent. Increasing the width $w_{s}$ over $150 \mathrm{~nm}$ has an undesired influence on the losses of the device [See Fig. 4(b)], which is why we choose the 150-nm-wide silicon strip which yields an almost flat wavelength response with a small penalty in insertion losses. The solid lines in Fig. 3 show the performance of the optimized PBS design with a tilt angle $\theta=$ $40^{\circ}$ and a silicon strip width $w_{s}=150 \mathrm{~nm}$, revealing an ER higher than $20 \mathrm{~dB}$ over an $86 \mathrm{~nm}$ bandwidth for both polarizations with IL lower than $1 \mathrm{~dB}$. The input tapers and the coupling region are 8 periods and 27 periods long, respectively, resulting in a device length of only $14 \mu \mathrm{m}$.
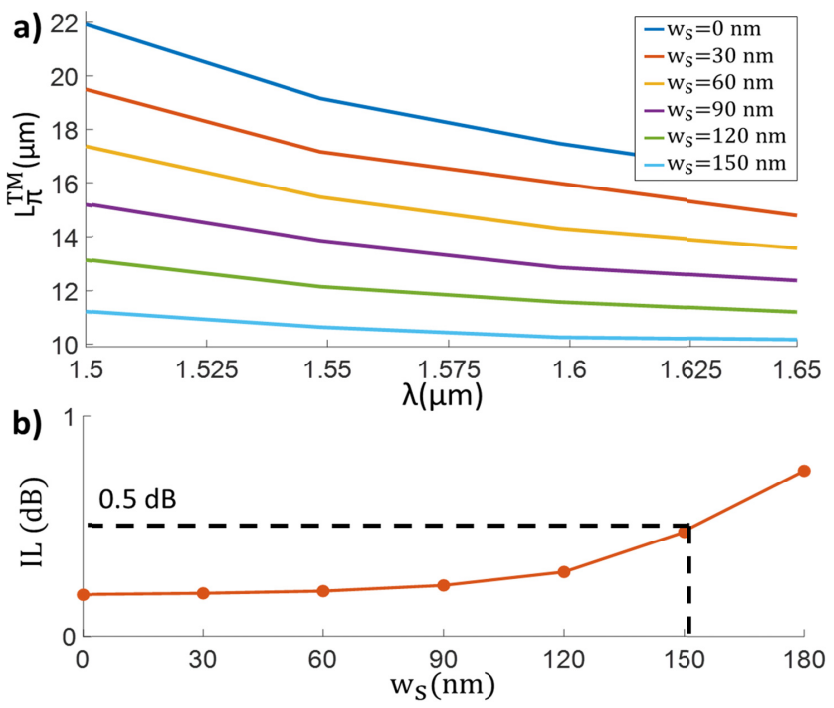

Fig. 4. TM polarization (a) coupling length and (b) insertion loss, of the tilted SWG directional couplers with different silicon strip widths.

The device has been fabricated in an open-access commercial 220-nm silicon-on-insulator platform via e-beam lithography with a 2- $\mu$ m-thick buried oxide (BOX) and a 2.2- $\mu$ m-thick SiO2 upper cladding [42]. In our measurement setup light from a tunable laser is coupled into a lensed polarization maintaining fiber. The fiber is mounted on a rotary stage, which, together with a Glan-Thompson polarizer, is used to achieve horizontal (TE) and vertical (TM) polarization states with a high extinction ratio. The light from the lensed fiber is coupled into the chip by using an SWG edge coupler with high and broadband coupling efficiency for both polarizations [43]. At the output, light is focused onto a photodetector via a microscope objective, with negligible crosstalk between the output ports which are separated by $30 \mu \mathrm{m}$. The fabricated device is characterized by measuring the power in both output ports (bar and cross) when the device is excited through the input port. Each device is excited first with TE polarized waveguide mode and then with TM mode. To determine the insertion loss, we use a reference waveguide to calibrate the setup and fiber-to-chip coupling losses for both polarizations. We then calculate the polarization extinction ratio by dividing the power in both output ports [see Eq. (2)]. In Figs. 5(a) and 5(b), we show the measured polarization extinction ratio and insertion loss of the fabricated device for both polarizations after applying the minimum phase technique [44]. The fabricated nominal device achieves an extinction ratio in excess of $15 \mathrm{~dB}$ and an insertion loss below $1 \mathrm{~dB}$ for both polarizations over a $72 \mathrm{~nm}$ bandwidth $(1505$ $\mathrm{nm}-1577 \mathrm{~nm}$ ). Figure 5(a) indicates that for long wavelengths the device performance is limited by the extinction ratio for TE polarization. Operation at longer wavelengths can be achieved by increasing the duty-cycle of the SWG structure, to enhance the modal confinement, which in turn suppresses the coupling of the TE mode to the cross port. Note that, for TM polarization, this increase in duty-cycle will only result in a minor detuning of the phase matching condition. In Figs. 5(c) and 5(d), we show the measured device performance when the duty cycle is increased from $50 \%$ to $60 \%$, i.e. the silicon blocks are $28 \mathrm{~nm}$ longer, and the overall device length is enlarged two periods. This biased device this yields insertion loss below $1 \mathrm{~dB}$ and extinction ratio in excess of $15 \mathrm{~dB}$ at longer wavelengths, from $1534 \mathrm{~nm}$ to $1601 \mathrm{~nm}$, i.e. a 67 $\mathrm{nm}$ bandwidth. Note that for applications incoherent optical 

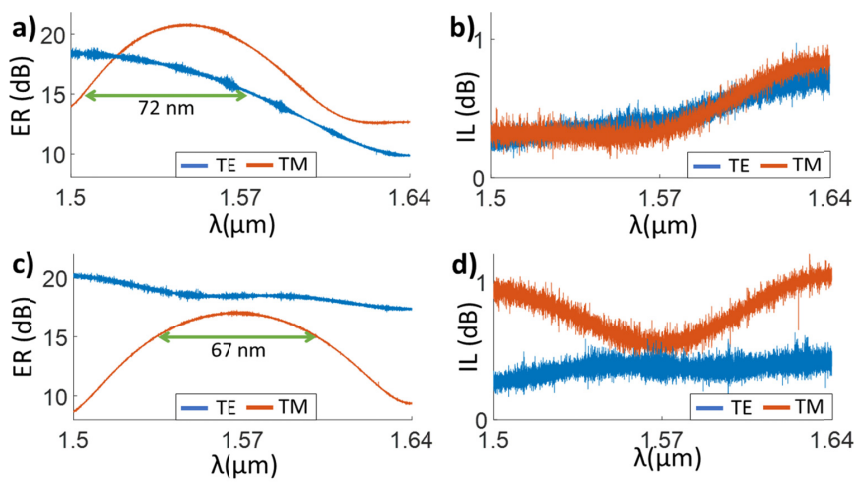

Fig. 5. Measured (a) extinction ratio and (b) insertion loss of the nominal device. The IL is below $1 \mathrm{~dB}$ and the ER is better than $15 \mathrm{~dB}$ in bandwidth of $72 \mathrm{~nm}$. Measured (c) extinction ratio and (d) insertion loss for a device with a duty cycle increased by $10 \%$. The IL are $<1 \mathrm{~dB}$ and the extinction ratio is $>15 \mathrm{~dB}$ in a bandwidth of $67 \mathrm{~nm}$.

receivers these specifications result in a power penalty of less than $1.5 \mathrm{~dB}$ [5].

In conclusion, we have proposed and experimentally demonstrated a new type of directional coupler based polarization beam splitter, which uses tilted subwavelength segments to control the symmetry between the coupler arms. The device exhibits a comparatively large minimum feature size of $100 \mathrm{~nm}$ and can be fabricated in a single etch step. The low insertion loss of the device, lower that $1 \mathrm{~dB}$ over the $143 \mathrm{~nm}$ measured bandwidth, place the device as an ideal component for polarization multiplexed coherent receivers. We believe that this new concept of refractive index symmetry engineering using tilted subwavelength gratings will open promising venues for polarization control in integrated optical devices.

Funding. Universidad de Málaga, Ministerio de Economía y Competitividad (MINECO) (TEC2016-80718-R), Ministerio de Educación, Cultura y Deporte (MECD) (FPU16/06762), and Fondo Europeo de Desarrollo Regional-FEDER, Proyecto I+D+i en el marco del Programa Operativo FEDER Andalucía 2014-2020 (UMA18-FEDERJA-219) and Alcyon Photonics (8.06/5.59.5045).

Disclosures. JMLG: Alcyon Photonics S.L. (F, P), AHB: Alcyon Photonics S.L. (F, P), AOM: Alcyon Photonics S.L. (F, P), AVV: Alcyon Photonics S.L. (F, I, P), IMF: Alcyon Photonics S.L. (F, P) RH: Alcyon Photonics S.L. (F, P).

\section{REFERENCES}

1. R. Soref, IEEE J. Sel. Top. Quantum Electron. 12, 1678 (2006).

2. T. Barwicz, M. R. Watts, M. A. Popović, P. T. Rakich, L. Socci, F. X. Kärtner, E. P. Ippen, and H. I. Smith, Nat. Photonics 1, 57 (2007).

3. H. Fukuda, K. Yamada, T. Tsuchizawa, T. Watanabe, H. Shinojima, and S. Itabashi, Opt. Express 16, 4872 (2008).

4. D. Dai, J. Bauters, and J. E. Bowers, Light Sci. Appl. 1, e1 (2012).

5. P. J. Reyes-Iglesias, I. Molina-Fernández, and A. Ortega-Moñux, Opt. Express 23, 12784 (2015).

6. A. J. Zilkie, P. Srinivasan, A. Trita, T. Schrans, G. Yu, J. Byrd, D. A. Nelson, K. Muth, D. Lerose, M. Alalusi, K. Masuda, M. Ziebell, H. Abediasl, J. Drake, G. Miller, H. Nykanen, E. Kho, Y. Liu, H. Liang, H. Yang, F. H. Peters, A. S. Nagra, and A. G. Rickman, IEEE J. Sel. Top. Quantum Electron. 25, 1 (2019).

7. T. Aalto, M. Cherchi, M. Harjanne, S. Bhat, P. Heimala, F. Sun, M. Kapulainen, T. Hassinen, and T. Vehmas, IEEE J. Sel. Top. Quantum Electron. 25, 1 (2019).
8. D. Taillaert, Harold Chong, P. I. Borel, L. H. Frandsen, R. M. De La Rue, and R. Baets, IEEE Photonics Technol. Lett. 15, 1249 (2003).

9. Y. Tang, D. Dai, and S. He, IEEE Photonics Technol. Lett. 21, 242 (2009).

10. J. M. Hong, H. H. Ryu, S. R. Park, J. W. Jeong, S. G. Lee, E.-H. Lee, S.-G. Park, D. Woo, S. Kim, and B.-H. O, IEEE Photonics Technol. Lett. 15, 72 (2003).

11. W. Jiang, X. Sun, and B. M. Azizur Rahman, Appl. Opt. 56, 2119 (2017).

12. M. R. Watts, H. A. Haus, and E. P. Ippen, Opt. Lett. 30, 967 (2005).

13. Y. Yin, Z. Li, and D. Dai, J. Light. Technol. 35, 2227 (2017).

14. B. Shen, P. Wang, R. Polson, and R. Menon, Nat. Photonics 9, 378 (2015).

15. Y. Liu, S. Wang, Y. Wang, W. Liu, H. Xie, Y. Yao, Q. Song, X. Zhang, Y. Yu, and K. Xu, Opt. Lett. 44, 4495 (2019).

16. D. Dai and J. E. Bowers, Opt. Express 19, 10940 (2011).

17. J. Wang, D. Liang, Y. Tang, D. Dai, and J. E. Bowers, Opt. Lett. 38, 4 (2013).

18. K. Chen, K. Yu, and S. He, IEEE Photonics J. 11, 1 (2019).

19. Y. Xiong, D.-X. Xu, J. H. Schmid, P. Cheben, S. Janz, and W. N. Ye, Opt. Express 22, 17458 (2014).

20. N.-C. Cheng, Y.-F. Ma, P.-H. Fu, C.-C. Chin, and D.-W. Huang, Appl. Opt. 54, 436 (2015)

21. J. Chee, S. Zhu, and G. Q. Lo, Opt. Express 20, 25345 (2012).

22. T. Zhang, X. Yin, L. Chen, and X. Li, Opt. Lett. 41, 356 (2016).

23. L. Liu, Q. Deng, and Z. Zhou, Opt. Lett. 41, 5126 (2016).

24. F. Zhang, H. Yun, Y. Wang, Z. Lu, L. Chrostowski, and N. A. F. Jaeger, Opt. Lett. 42, 235 (2017).

25. B. Ni and J. Xiao, IEEE J. Quantum Electron. 53, 1 (2017)

26. Y. Tian, J. Qiu, C. Liu, S. Tian, Z. Huang, and J. Wu, Opt. Express 27, 999 (2019).

27. P. Cheben, D.-X. Xu, S. Janz, and A. Densmore, Opt. Express 14, 4695 (2006).

28. R. Halir, A. Ortega-Moñux, D. Benedikovic, G. Z. Mashanovich, J. G. Wangüemert-Perez, J. H. Schmid, Í. Molina-Fernandez, and P. Cheben, Proc. IEEE 106, 2144 (2018).

29. P. Cheben, R. Halir, J. H. Schmid, H. A. Atwater, and D. R. Smith, Nature 560,565 (2018).

30. J. M. Luque-González, R. Halir, J. G. Wangüemert-Pérez, J. De-OlivaRubio, J. H. Schmid, P. Cheben, Í. Molina-Fernández, and A. OrtegaMoñux, Laser Photon. Rev. 13, 1900172 (2019).

31. C. Li and D. Dai, Opt. Lett. 42, 4243 (2017).

32. T. Huang, Y. Xie, Y. Wu, Z. Cheng, S. Zeng, and P. S. Ping, Appl. Opt. 58, 2264 (2019).

33. J. M. Luque-González, A. Herrero-Bermello, A. Ortega-Moñux, í. Molina-Fernández, A. V. Velasco, P. Cheben, J. H. Schmid, S. Wang, and R. Halir, Opt. Lett. 43, 4691 (2018).

34. A. Herrero Bermello, J. M. Luque González, A. Velasco, A. OrtegaMoñux, P. Cheben, and R. Halir, IEEE Photonics J. 11, 1 (2019).

35. J. D. Sarmiento-Merenguel, A. Ortega-Moñux, J.-M. Fédéli, J. G. Wangüemert-Pérez, C. Alonso-Ramos, E. Durán-Valdeiglesias, P. Cheben, Í. Molina-Fernández, and R. Halir, Opt. Lett. 41, 3443 (2016).

36. A. Ortega-Moñux, J. Čtyroký, P. Cheben, J. H. Schmid, S. Wang, Í. Molina-Fernández, and R. Halir, Opt. Express 25, 12222 (2017).

37. C.-L. Chen, Foundations for Guided-Wave Optics (John Wiley \& Sons, Inc., 2006).

38. "FULLWAVE FDTD Simulation Software," https://www.synopsys.com/optical-solutions/rsoft/passive-devicefullwave.html.

39. J. Gonzalo Wangüemert-Pérez, P. Cheben, A. Ortega-Moñux, C. Alonso-Ramos, D. Pérez-Galacho, R. Halir, Í. Molina-Fernández, D. Xu, and J. H. Schmid, Opt. Lett. 39, 4442 (2014).

40. D. W. Kim, M. H. Lee, Y. Kim, and K. H. Kim, Opt. Express 23, 998 (2015).

41. Y. Kim, M. H. Lee, Y. Kim, and K. H. Kim, Opt. Lett. 43, 3241 (2018).

42. "Applied Nanotools Inc. Canada," https://www.appliednt.com/nanosoi/.

43. P. Cheben, J. H. Schmid, S. Wang, D. Xu, M. Vachon, S. Janz, J. Lapointe, Y. Painchaud, and M. Picard, Opt. Express 23, 22553 (2015).

44. R. Halir, Í. Molina-Fernández, J. G. Wangüemert-Pérez, A. OrtegaMoñux, J. De-Oliva-Rubio, and P. Cheben, Opt. Express 17, 8349 (2009). 


\section{FULL REFERENCES}

1. R. Soref, "The Past, Present, and Future of Silicon Photonics," IEEE J. Sel. Top. Quantum Electron. 12, 1678 (2006).

2. T. Barwicz, M. R. Watts, M. A. Popović, P. T. Rakich, L. Socci, F. X. Kärtner, E. P. Ippen, and H. I. Smith, "Polarization-transparent microphotonic devices in the strong confinement limit," Nat. Photonics 1, 57 (2007).

3. H. Fukuda, K. Yamada, T. Tsuchizawa, T. Watanabe, H. Shinojima, and S. Itabashi, "Silicon photonic circuit with polarization diversity," Opt. Express 16, 4872 (2008).

4. D. Dai, J. Bauters, and J. E. Bowers, "Passive technologies for future large-scale photonic integrated circuits on silicon: polarization handling, light non-reciprocity and loss reduction," Light Sci. Appl. 1, e1 (2012).

5. P. J. Reyes-Iglesias, í. Molina-Fernández, and A. Ortega-Moñux, "Sensitivity penalty induced by non-ideal dual polarization downconverter in digital coherent receivers," Opt. Express 23, 12784 (2015).

6. A. J. Zilkie, P. Srinivasan, A. Trita, T. Schrans, G. Yu, J. Byrd, D. A. Nelson, K. Muth, D. Lerose, M. Alalusi, K. Masuda, M. Ziebell, H. Abediasl, J. Drake, G. Miller, H. Nykanen, E. Kho, Y. Liu, H. Liang, H. Yang, F. H. Peters, A. S. Nagra, and A. G. Rickman, "Multi-Micron Silicon Photonics Platform for Highly Manufacturable and Versatile Photonic Integrated Circuits," IEEE J. Sel. Top. Quantum Electron. 25, 1 (2019).

7. T. Aalto, M. Cherchi, M. Harjanne, S. Bhat, P. Heimala, F. Sun, M. Kapulainen, T. Hassinen, and T. Vehmas, "Open-Access 3- $\mu \mathrm{m}$ SOI Waveguide Platform for Dense Photonic Integrated Circuits," IEEE J. Sel. Top. Quantum Electron. 25, 1 (2019).

8. D. Taillaert, Harold Chong, P. I. Borel, L. H. Frandsen, R. M. De La Rue, and R. Baets, "A compact two-dimensional grating coupler used as a polarization splitter," IEEE Photonics Technol. Lett. 15, 1249 (2003).

9. Y. Tang, D. Dai, and S. He, "Proposal for a grating waveguide serving as both a polarization splitter and an efficient coupler for silicon-oninsulator nanophotonic circuits," IEEE Photonics Technol. Lett. 21, 242 (2009).

10. J. M. Hong, H. H. Ryu, S. R. Park, J. W. Jeong, S. G. Lee, E.-H. Lee, S.-G. Park, D. Woo, S. Kim, and B.-H. O, "Design and fabrication of a significantly shortened multimode interference coupler for polarization splitter application," IEEE Photonics Technol. Lett. 15, 72 (2003).

11. W. Jiang, X. Sun, and B. M. Azizur Rahman, "Compact and fabricationtolerant polarization splitter based on horizontal triple-slot waveguide," Appl. Opt. 56, 2119 (2017).

12. M. R. Watts, H. A. Haus, and E. P. Ippen, "Integrated mode-evolutionbased polarization splitter," Opt. Lett. 30, 967 (2005).

13. Y. Yin, Z. Li, and D. Dai, "Ultra-Broadband Polarization Splitter-Rotator Based on the Mode Evolution in a Dual-Core Adiabatic Taper," J. Light. Technol. 35, 2227 (2017).

14. B. Shen, P. Wang, R. Polson, and R. Menon, "An integratednanophotonics polarization beamsplitter with $2.4 \times 2.4 \mu \mathrm{m}^{2}$ footprint," Nat. Photonics 9, 378 (2015).

15. Y. Liu, S. Wang, Y. Wang, W. Liu, H. Xie, Y. Yao, Q. Song, X. Zhang, Y. Yu, and $\mathrm{K} . \mathrm{Xu}$, "Subwavelength polarization splitter-rotator with ultracompact footprint," Opt. Lett. 44, 4495 (2019).

16. D. Dai and J. E. Bowers, "Novel concept for ultracompact polarization splitter-rotator based on silicon nanowires," Opt. Express 19, 10940 (2011).

17. J. Wang, D. Liang, Y. Tang, D. Dai, and J. E. Bowers, "Realization of an ultra-short silicon polarization beam splitter with an asymmetrical bent directional coupler," Opt. Lett. 38, 4 (2013).

18. Y. Xiong, D.-X. Xu, J. H. Schmid, P. Cheben, S. Janz, and W. N. Ye, "Fabrication tolerant and broadband polarization splitter and rotator based on a taper-etched directional coupler," Opt. Express 22, 17458 (2014).

19. N.-C. Cheng, Y.-F. Ma, P.-H. Fu, C.-C. Chin, and D.-W. Huang, "Horizontal slot waveguides for polarization branching control," Appl. Opt. 54, 436 (2015).
20. J. Chee, S. Zhu, and G. Q. Lo, "CMOS compatible polarization splitter using hybrid plasmonic waveguide," Opt. Express 20, 25345 (2012).

21. T. Zhang, X. Yin, L. Chen, and X. Li, "Ultra-compact polarization beam splitter utilizing a graphene-based asymmetrical directional coupler," Opt. Lett. 41, 356 (2016).

22. L. Liu, Q. Deng, and Z. Zhou, "Manipulation of beat length and wavelength dependence of a polarization beam splitter using a subwavelength grating," Opt. Lett. 41, 5126 (2016).

23. F. Zhang, H. Yun, Y. Wang, Z. Lu, L. Chrostowski, and N. A. F. Jaeger, "Compact broadband polarization beam splitter using a symmetric directional coupler with sinusoidal bends," Opt. Lett. 42, 235 (2017).

24. B. Ni and J. Xiao, "Ultracompact and Broadband Silicon-Based Polarization Beam Splitter Using an Asymmetrical Directional Coupler," IEEE J. Quantum Electron. 53, 1 (2017).

25. Y. Tian, J. Qiu, C. Liu, S. Tian, Z. Huang, and J. Wu, "Compact polarization beam splitter with a high extinction ratio over $\mathrm{S}+\mathrm{C}+\mathrm{L}$ band," Opt. Express 27, 999 (2019).

26. K. Chen, K. Yu, and S. He, "High Performance Polarization Beam Splitter Based on Cascaded Directional Couplers Assisted by Effectively Anisotropic Structures," IEEE Photonics J. 11, 1 (2019).

27. P. Cheben, D.-X. Xu, S. Janz, and A. Densmore, "Subwavelength waveguide grating for mode conversion and light coupling in integrated optics," Opt. Express 14, 4695 (2006).

28. R. Halir, A. Ortega-Moñux, D. Benedikovic, G. Z. Mashanovich, J. G. Wangüemert-Perez, J. H. Schmid, I. Molina-Fernandez, and P. Cheben, "Subwavelength-Grating Metamaterial Structures for Silicon Photonic Devices," Proc. IEEE 106, 2144 (2018).

29. P. Cheben, R. Halir, J. H. Schmid, H. A. Atwater, and D. R. Smith, "Subwavelength integrated photonics," Nature 560, 565 (2018).

30. J. M. Luque-González, R. Halir, J. G. Wangüemert-Pérez, J. De-OlivaRubio, J. H. Schmid, P. Cheben, Í. Molina-Fernández, and A. OrtegaMoñux, "An Ultracompact GRIN-Lens-Based Spot Size Converter using Subwavelength Grating Metamaterials," Laser Photon. Rev. 13, 1900172 (2019).

31. C. Li and D. Dai, "Compact polarization beam splitter for silicon photonic integrated circuits with a $340-\mathrm{nm}$-thick silicon core layer," Opt. Lett. 42, 4243 (2017).

32. T. Huang, Y. Xie, Y. Wu, Z. Cheng, S. Zeng, and P. S. Ping, "Compact polarization beam splitter assisted by subwavelength grating in triplewaveguide directional coupler," Appl. Opt. 58, 2264 (2019).

33. J. M. Luque-González, A. Herrero-Bermello, A. Ortega-Moñux, í. Molina-Fernández, A. V. Velasco, P. Cheben, J. H. Schmid, S. Wang, and R. Halir, "Tilted subwavelength gratings: controlling anisotropy in metamaterial nanophotonic waveguides," Opt. Lett. 43, 4691 (2018).

34. A. Herrero Bermello, J. M. Luque González, A. Velasco, A. OrtegaMoñux, P. Cheben, and R. Halir, "Design of a broadband polarization splitter based on anisotropy-engineered tilted subwavelength gratings," IEEE Photonics J. 11, 1 (2019).

35. J. D. Sarmiento-Merenguel, A. Ortega-Moñux, J.-M. Fédéli, J. G. Wangüemert-Pérez, C. Alonso-Ramos, E. Durán-Valdeiglesias, P. Cheben, Í. Molina-Fernández, and R. Halir, "Controlling leakage losses in subwavelength grating silicon metamaterial waveguides," Opt. Lett. 41,3443 (2016).

36. A. Ortega-Moñux, J. Čtyroký, P. Cheben, J. H. Schmid, S. Wang, í. Molina-Fernández, and R. Halir, "Disorder effects in subwavelength grating metamaterial waveguides," Opt. Express 25, 12222 (2017).

37. C.-L. Chen, Foundations for Guided-Wave Optics (John Wiley \& Sons, Inc., 2006).

38. "FULLWAVE FDTD Simulation Software," https://www.synopsys.com/optical-solutions/rsoft/passive-devicefullwave.html.

39. J. Gonzalo Wangüemert-Pérez, P. Cheben, A. Ortega-Moñux, C. Alonso-Ramos, D. Pérez-Galacho, R. Halir, I. Molina-Fernández, D. Xu, and J. H. Schmid, "Evanescent field waveguide sensing with subwavelength grating structures in silicon-on-insulator," Opt. Lett. 39, 4442 (2014).

40. D. W. Kim, M. H. Lee, Y. Kim, and K. H. Kim, "Planar-type polarization beam splitter based on a bridged silicon waveguide coupler," Opt. Express 23, 998 (2015).

41. Y. Kim, M. H. Lee, Y. Kim, and K. H. Kim, "High-extinction-ratio 
directional-coupler-type polarization beam splitter with a bridged silicon wire waveguide," Opt. Lett. 43, 3241 (2018).

42. "Applied Nanotools

Inc.

Canada," https://www.appliednt.com/nanosoi/.

43. P. Cheben, J. H. Schmid, S. Wang, D. Xu, M. Vachon, S. Janz, J. Lapointe, Y. Painchaud, and M. Picard, "Broadband polarization independent nanophotonic coupler for silicon waveguides with ultrahigh efficiency," Opt. Express 23, 22553 (2015).

44. R. Halir, Í. Molina-Fernández, J. G. Wangüemert-Pérez, A. OrtegaMoñux, J. De-Oliva-Rubio, and P. Cheben, "Characterization of integrated photonic devices with minimum phase technique," Opt. Express 17, 8349 (2009). 